\title{
Nematodes Follow a Leader
}

\author{
Hilal Erdogan ${ }^{1,2 t}$, Karin Cruzado-Gutierrez ${ }^{1 \dagger}$, Glen Stevens ${ }^{1}$, David Shapiro-Ilan ${ }^{3}$, \\ Fatma Kaplan ${ }^{4}$, Hans Alborn ${ }^{5}$ and Edwin Lewis ${ }^{1 *}$ \\ ${ }^{1}$ Department of Entomology, Plant Pathology and Nematology, University of Idaho, Moscow, ID, United States, ${ }^{2}$ Faculty \\ of Agriculture, Department of Biosystems Engineering, Bursa Uludağ University, Bursa, Turkey, ${ }^{3}$ USDA-ARS, SEFTNRL, \\ Byron, GA, United States, ${ }^{4}$ Pheronym Inc., Davis, CA, United States, ${ }^{5}$ Center for Medical, Agricultural and Veterinary \\ Entomology (CMAVE), Southeast Area (SEA), United States Department of Agriculture-Agricultural Research Service \\ (USDA-ARS), Gainesville, FL, United States
}

\section{OPEN ACCESS}

Edited by:

Ralf J. Sommer,

Max Planck Institute for Developmental Biology, Max Planck Society (MPG), Germany

Reviewed by:

Christine Griffin,

Maynooth University, Ireland Adler Ray Dillman,

University of California, Riverside, United States

*Correspondence: Edwin Lewis

eelewis@uidaho.edu

†These authors share first authorship

Specialty section:

This article was submitted to Behavioral and Evolutionary Ecology, a section of the journa Frontiers in Ecology and Evolution

Received: 12 July 2021 Accepted: 15 October 2021 Published: 04 November 2021

Citation:

Erdogan H, Cruzado-Gutierrez K Stevens G, Shapiro-llan D, Kaplan F, Alborn H and Lewis E (2021) Nematodes Follow a Leader. Front. Ecol. Evol. 9:740351. doi: 10.3389/fevo.2021.740351
Aggregated movement and population structure are known in entomopathogenic nematodes, which are obligate insect parasites. Aggregation behavior in the absence of external stimuli suggests communication among individuals, often in the form of trailfollowing, which has not been shown by nematodes of any kind. Interactions among individuals are an essential basis of following behaviors and can have significant fitness consequences. We explored intraspecific and interspecific interactions among three Steinernema species (S. glaseri, S. carpocapsae, and S. feltiae) in terms of trail following, and fitness outcomes of following heterospecific individuals. We found that the following behavior is context dependent. Following behavior among conspecifics was significantly increased when the lead nematode had prior contact with host cuticle. However, we did not find a clear association between the following response to heterospecific IJs and their reproductive success in a co-infected host.

\section{Keywords: nematode, trail following, aggregation, mass-attack, parasite, infection}

\section{INTRODUCTION}

Group behaviors are widespread among animals and are hypothesized to offer fitness benefits to individuals that would be unavailable if they were solitary. Group behaviors usually involve individual responses to various stimuli that could be associated with resources, environmental conditions, or other members of the group. One such group behavior is trail following. Trailfollowing behaviors can be driven by such disparate variables as pheromones (Gehlbach et al., 1971; Traniello, 1982) and fluid dynamics (Dehnhardt et al., 2001; Fourcassié et al., 2010). Here we document trail following behavior by nematodes. While nematodes and arthropods comprise the superphylum Ecdysozoa, and there is a vast literature regarding trail following by arthropods (Traniello, 1982; Reinhard and Kaib, 2001; Fourcassié et al., 2010), no examples are known of following behaviors within the phylum Nematoda, despite the often-aggregated population structures of most species. Following behavior can have significant fitness consequences when groups of conspecifics can garner resources that singletons cannot (e.g., mass attack by parasites).

Entomopathogenic nematodes (EPN) are well-studied due to their potential as biological control agents of insect pests and their use as biological models (Stock, 2005; McMullen et al., 2017). In nature, they are primarily parasites of insects (Kaya and Gaugler, 1993; Lewis et al., 2006). Infective juveniles (IJs), the specialized third-stage juvenile (analogous to the dauer stage of Caenorhabditis elegans), are the only life stage found outside the host and its single function is to infect a new host. After finding a host, IJs enter the hemocoel and release their symbiotic bacteria (Xenorhabdus spp. 
for Steinernema spp. EPN). An infected host generally dies within 24-36 h due to septicemia or toxemia (Lewis et al., 2006). EPNs resume development and feed on the bacteria, reproduce for 1-3 generations, and IJs develop in response to a number of cues within the cadaver (Kaya and Gaugler, 1993; Lewis et al., 1996). IJs emerge, disperse and forage for a new host (Lewis et al., 1992, 2006).

IJ host finding behaviors are strongly influenced by chemical signals (e.g., ascaroside pheromones) and other chemical cues (e.g., $\mathrm{CO}_{2}$ and damage induced root volatiles) (Lewis et al., 1992; Shapiro and Glazer, 1996; Ali et al., 2012; ShapiroIlan et al., 2014, 2019; Kaplan et al., 2020). EPNs exhibit the aggregated spatial distribution common to nematodes in natural populations (Lawrence et al., 2006; Spiridonov et al., 2007; Campos-Herrera et al., 2011) and laboratory and field trials (Shapiro-Ilan et al., 2014; Ruan et al., 2018). Presumably, a patchy distribution is reinforced by the need for mass attack to successfully overcome the host immune system plus the mass emergence of IJs from a single infected host $(50,000+$ IJs are often produced), their limited capacity for movement, and the heightened attractiveness of hosts infected by conspecifics (Fushing et al., 2008). Aggregative patterns have been observed in the presence and the absence of external cues (Lewis et al., 1992, 2006; Shapiro-Ilan et al., 2014).

Aggregations are by-products of the behaviors of individuals. Association with conspecifics in the absence of external stimuli (Ruan et al., 2018) suggests communication among individuals, as demonstrated by other organisms such as ants and termites; foraging individuals leave a trail during foraging which can be followed by other individuals (Reinhard and Kaib, 2001; Fourcassié et al., 2010) and thereby provide benefits to members of the group. Factors such as contact with the cuticle of a potential host alters IJ foraging behavior (Lewis et al., 1992), and thus EPN IJs would benefit from following the trail of an IJ, particularly one that had made contact with a host. Most studies of EPN host finding are conducted with large groups of IJs and data are reported as the percentage of individuals responding to a cue such as the presence of a host (e.g., Lewis et al., 1995a,b; Shapiro-Ilan et al., 2014). But how many IJs are responding to host cues and how many are responding to and following other IJs instead of or in addition to responding to cues? Willett et al. (2017) showed that groups of naïve IJs followed other IJs that had been exposed to host cues, but they did not examine behavioral responses of individual EPN IJs. Measuring behavioral responses of EPN at the scale of an individual worm is required to understand this behavior.

Interactions among individuals of parasitic species are not limited to colonizing a host; the ability to overcome competitors to gain resources is also important. An insect host can be infected by multiple EPN species (Alatorrre-Rosas and Kaya, 1990; Lewis et al., 2006) and interspecific interactions between sympatric species occur (Mrácek et al., 2005). Differential responses to hosts infected by heterospecific nematodes have been recorded and related to reproductive success (Koppenhofer et al., 1995; Wang and Ishibashi, 1999; Puza and Mrácek, 2009). For example, the reproduction of Steinernema carpocapsae is reduced when Steinernema glaseri co-infects a host (Koppenhofer et al., 1995).
Such competitive outcomes related to reproductive fitness should impact the behaviors of individuals in ways that reduce the odds of losing a competitive interaction. But how host choice is impacted by infection status has only been explored for conspecific interactions.

Given our interest in understanding basic behaviors in these worms, we conducted a series of experiments to assess following behavior in EPN. Using a standard agar-plate arena, we examined con- and heterospecific following behaviors among a group of three EPN species from the genus Steinernema. We hypothesized that these species would exhibit following behavior that is context dependent. Specifically, we predicted (1) that IJs would follow conspecifics, and that (2) conspecific following would be increased if the leader had prior host contact. We also predicted that (3) IJ following of heterospecific nematodes would be influenced by the outcome of competition within the infected host.

\section{MATERIALS AND METHODS}

\section{Nematodes}

Steinernema glaseri (SG strain), Steinernema feltiae (SN strain), and Steinernema carpocapsae (All strain) were propagated by exposing last instar Galleria mellonella to $50 \mathrm{IJs}$ at $25^{\circ} \mathrm{C}$ on Whatman filter paper. After death, G. mellonella were placed onto White traps (White, 1927) for IJ collection. Briefly, infected cadavers are placed into a $60 \mathrm{~mm}$ diameter Petri dish lined with moistened filter paper and this dish is floated on distilled water in a $90 \mathrm{~mm}$ diameter Petri dish. When IJs emerge, they leave the smaller dish and are entrapped in the water. IJs were stored in distilled water at $14^{\circ} \mathrm{C}$ and used in experiments within 15 days of harvest.

\section{Con- and Hetero-Specific Following Experiments \\ General Methods}

Trail following experiments were conducted to evaluate IJ responses to trails made by previous IJs. These experiments were conducted in an agar plate arena following methods from Dalzell et al. (2011). Briefly, $60 \mathrm{~mm}$ dia. glass Petri plates (Corning ${ }^{\circledR}$, NY, United States) were filled with approximately $15 \mathrm{ml}$ of $2 \%$ water agar (Fisher Scientific, NJ, United States) (Figure 1). A cylindrical plastic bar $(6 \mathrm{~mm}$ diam. $\times 20 \mathrm{~mm}$ length) was placed on the center of each plate before the agar began to set to create a canal. Bars were removed from the agar after 30-40 min, leaving a semicylindrical depression in the surface. To verify the length and to set the center point of the canal, a line was drawn on the underside of the agar plates. After removal of bars, wells were made at each end of the canal using a metal cork borer $(9.5 \mathrm{~mm}$ in external diameter; $8.0 \mathrm{~mm}$ inner diameter). See Figure 1 for further details. Plates were covered after $30 \mathrm{~min}$ and stored at $22^{\circ} \mathrm{C}$ until use.

Before each assay, IJs were concentrated on filter paper (25 $\mathrm{mm}$ in diameter P8, Fisher Scientific, Pittsburgh, PA, United States) by vacuum filtration, after which the filter paper containing IJs was inverted on a $2 \%$ water agar surface in a 


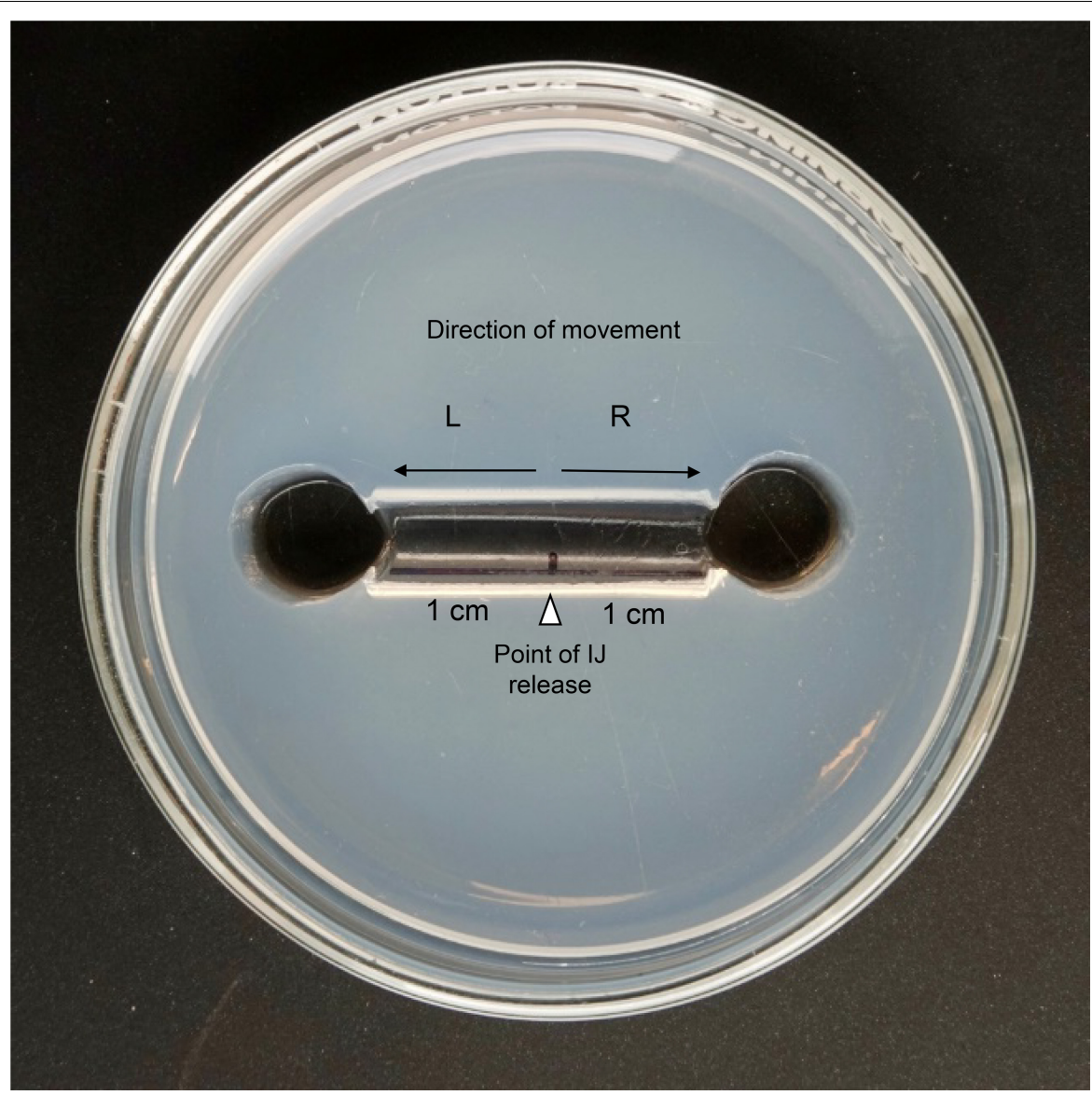

FIGURE 1 Agar plate arena following. Two wells are connected by a central canal and filled with 3 ml of deionized water (DI) to balance the moisture of plates through the experiment. After $15 \mathrm{~min}, 2 \mathrm{ml}$ of DI water was removed from plates, leaving a thin layer of water into the canal. The remaining thin layer of DI is necessary to keep infective juveniles (IJs) in the canal throughout the experiment and to diffuse cues. The flat level surface was verified by a bubble level cell phone application. A single infective juvenile $(\mathrm{IJ})$ was released into the middle of the central channel in the assay arena. After 10 min, the direction of movement of each IJ was recorded as right $(R)$ or left $(L)$ based on the movement of the IJ from the release point. Any IJ movement away from the point of introduction was considered as directional migration.

$9 \mathrm{~cm}$ diameter Petri dish. IJs were maintained on the agar surface for approximately $4 \mathrm{~h}$. For the lead IJ, individuals were picked from the agar with an autoclaved wooden toothpick or metal lab probe for each assay. Lead IJs were observed continuously for $10 \mathrm{~min}$; if no movement occurred after $10 \mathrm{~min}$, the IJ and plate were discarded. Any IJ that moved a body length or more from the center line introduction point was considered to have moved; the distance of each IJs traveling in the canal was not recorded. To avoid movement bias, the surface was leveled using a bubble level and handling and moving the plates were minimized. Any plates which had an accidental movement were discarded and not scored.

\section{Exposure to Host Cuticle}

Lead IJs were either exposed to G. mellonella cuticle for $30 \mathrm{~min}$ (host exposure treatment) or not exposed immediately prior to their release into the arena. To expose IJs to G. mellonella cuticle, larvae were killed by freezing and then allowed to warm to room temperature. Once larvae reached room temperature they were placed on moistened filter paper inside a $60 \mathrm{~mm}$ Petri dish (Lewis et al., 1992). IJs were concentrated on filter paper by vacuum and transferred to the $G$. mellonella cuticle with an autoclaved wooden toothpick or metal laboratory probe. After $30 \mathrm{~min}$ of exposure to cuticle at room temperature $\left(21^{\circ} \mathrm{C}\right)$, IJs were picked individually from G. mellonella cuticle and placed directly on the center of the canal (above the marked line, Figure 1) in the assay arena. To minimize chances for contamination we used a freshly sterilized tool every time an IJ was transferred.

\section{Conspecific and Heterospecific Pairings}

For conspecific assays the lead IJ and followers belonged to the same species. For heterospecific assays the lead IJ belonged to one of the selected species and the second, third, fourth IJs were a different species.

\section{Scoring and Data Collection}

In conditions when the lead IJ left the central introduction point, its direction was noted after $10 \mathrm{~min}$, and it was removed from 
the arena using an autoclaved wooden toothpick or lab probe. A second IJ was released to the center of the canal using a new autoclaved wooden toothpick or lab probe. If the subsequent IJ left the central line introduction point by more than a body length, the movement of that IJ was scored as either toward (following) or away from (not following) the path of the lead IJ. The process was conducted three times at 10-min intervals for each lead IJ. Movement of IJs in the same direction as the lead one was scored as "following." If the second, third or fourth IJ did not move this was also recorded. In summary, the lead IJ was either exposed to host cuticle or not, and the subsequent IJs were either conspecific or heterospecific to the first IJ and never exposed to host cuticle. There were 15 repetitions (plates) per treatment, giving a total of $45 \mathrm{IJs}$ tested as potential followers, each of which was considered a replication. The scorings for conspecific and heterospecific pairings and cuticle exposure versus no exposure were conducted following the same procedure.

\section{Interspecific Competition}

Reproductive success (measured as mean IJ production/infected cadaver) of each species was compared by exposing IJs of either one or two species to a single G. mellonella larva. Comparisons were divided into two experimental groups; one group consisted of evaluating reproductive success when a $G$. mellonella larva was exposed to a single species (conspecific) and the other group evaluated IJ production when a G. mellonella larva was exposed to the same number of IJs with half of one species and half of another. This allowed us to assess the competitive strength of each species, which we predicted would be related to the propensity of IJs to follow heterospecific IJs.

In these assays, each G. mellonella larva was exposed to 60 IJs, either all of a single species, or 30 of one species and 30 of another, in an inoculation cocoon (Lewis et al., 1995b). The cocoon was made from filter paper $(55 \mathrm{~mm}$ dia. P8, Fisher Scientific, Pittsburgh, PA, United States) which was rolled into a cone. IJs were pipetted into a cocoon in $200 \mu \mathrm{l}$ of deionized water. One G. mellonella larva was placed into the cocoon and both ends were folded and covered with tape and placed inside a Petri dish. Cocoons were incubated at room temperature $\left(21^{\circ} \mathrm{C}\right)$ until the waxworms died, after which hosts were transferred into White traps. IJs were harvested from G. mellonella cadavers until they stopped emerging (minimum 14 days post-emergence). The number of IJs of each species that emerged from each cadaver was estimated by serial dilution. The experiment was conducted three times with five repetitions per treatment.

\section{Statistical Analysis \\ Following Experiments}

Following analyses were conducted using count data and binomial probability analysis. In keeping with our original predictions, the observed following and not following proportions were compared for each set of trials (i.e., for each bar in Figures 2, 3) to the "expected" following: not following ratio of 50:50.

During statistical analysis, we were required to decide how to classify IJs that did not move from the introduction point (i.e., whether to exclude them from analysis, or count them as "not following"). We chose to analyze the data both ways and report any major differences in interpretation between the two approaches.

\section{Competition and Reproduction}

The reproductive success of the different nematode species in competition was assessed using the number of IJs emerging from G. mellonella that had been placed in filter paper cocoons. We assessed victory two ways: (1) by recording when one species produced $90 \%$ or more of the total emerging IJs from a cadaver, and (2) by comparing the proportional IJ production of each species in mixed and single-species infections. In the first assessment of reproductive success, we tested whether a given species won significantly more than $50 \%$ of head-to-head matches using chi-square analysis. In the second assessment, proportional IJ production $\left(\mathrm{IJ}_{\mathrm{p}}\right)$ was calculated by dividing the number of IJs emerging from each cadaver in heterospecific treatments by the average number of IJs of the corresponding species' con-specific treatment. For a given species, the equation for $\mathrm{IJ}_{\mathrm{p}}$ would be the following:

$$
I J_{p}=\frac{\sum_{\text {het }_{i}=1}^{N}\left(I I_{h t_{i}} / \overline{I I_{c o n}}\right)}{N}
$$

Where $N$ is the number of replications per treatment, het $t_{i}$ is the number of IJs in the $i^{\text {th }}$ repetition per species in the mixed population, and $I J_{c o n}$ is the average number of IJs in conspecific controls. In this case, an $\mathrm{IJ}_{\mathrm{p}}$ value of 1 would indicate that a species tended to produce the same number of IJs in heterospecific and conspecific conditions; $\mathrm{IJ}_{\mathrm{p}}$ values below 1 indicate a suppression of IJ production when in competition. Means and variability of $\mathrm{IJ}_{\mathrm{p}}$ were compared across each species pairings to determine whether a particular species was most successful in competition in a given pairing. The null hypothesis was that the $\mathrm{IJ}_{\mathrm{p}}$ of the two species in a given species pairing would be the same; when one species significantly dominated the infections (at an alpha $<0.05$ ), the null hypothesis was rejected (Software SAS 9.4).

\section{RESULTS}

\section{Following Response}

\section{Conspecific Following}

When given a choice between following a conspecific IJ and going in the opposite direction, following occurred significantly more than $50 \%$ of the time when the lead IJ had been exposed to host cuticle $(P<0.001)$ in all species tested. Average rates of following after host contact in all species was approximately $80 \%$. Only S. glaseri followed significantly more than $50 \%$ of the time when the lead IJ had not been exposed to host cuticle (Figure 2).

During analysis, we were required to decide how to classify IJs that did not move from the introduction point (e.g., whether to exclude them from analysis, or count them as "not following"). We analyzed the data both ways, and there were no differences in the interpretation in the case of the conspecific following trials. 

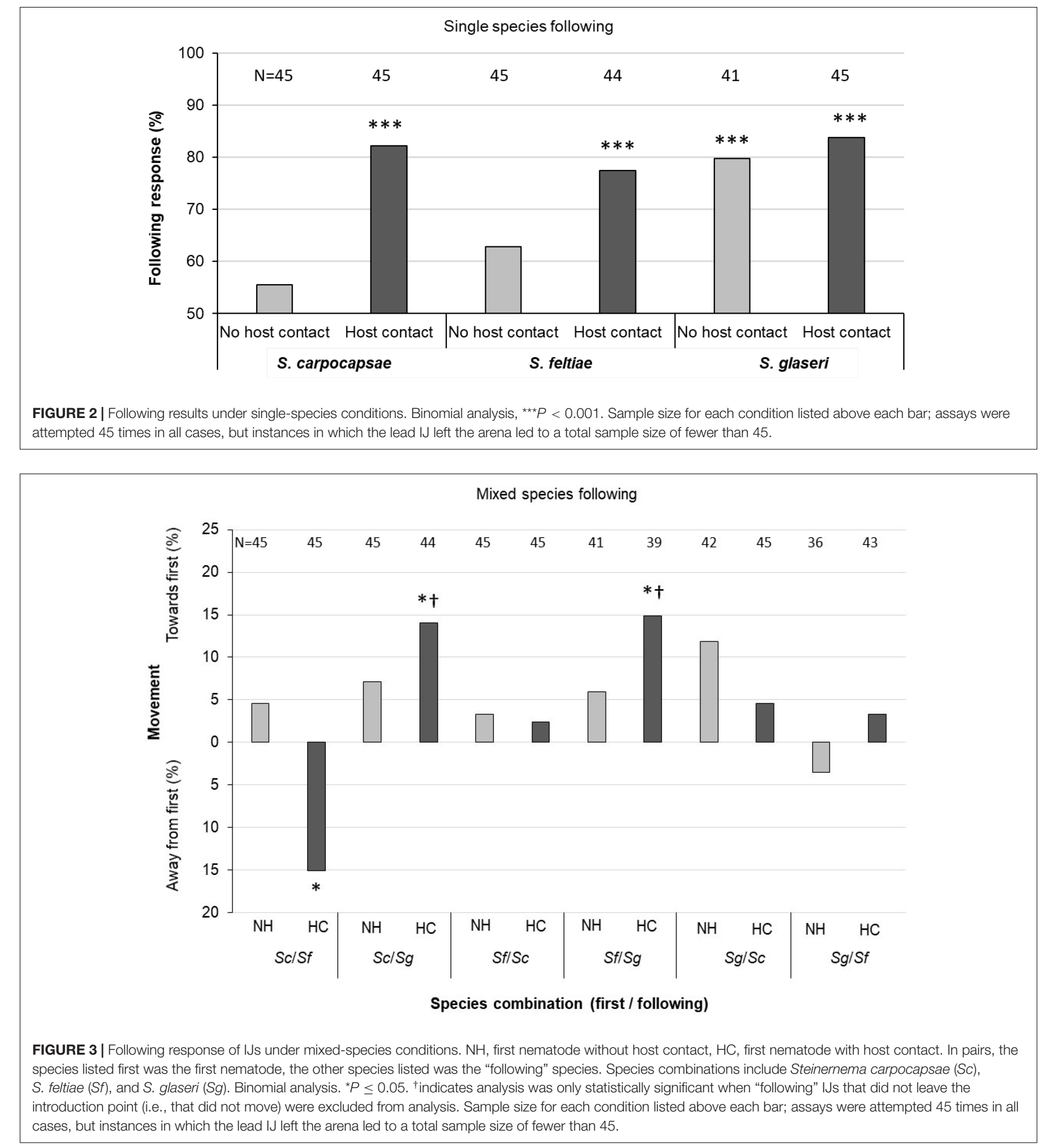

While the statistics changed subtly, all significance levels were maintained at $P<0.001$ or lower.

\section{Heterospecific Following}

The behavioral response of IJs to heterospecific leaders varied across the six species pairings, and in 3 of the 6 cases were significantly influenced by host contact of the lead IJ (Figure 3).
Interestingly, $S$. feltiae moved in the opposite direction when host-exposed $S$. carpocapsae was the leader $(P<0.05)$. In the other two significant cases, S. glaseri was more likely to follow either species if the lead IJ had host contact. S. carpocapsae did not significantly follow or avoid either S. glaseri or S. feltiae.

In the heterospecific analyses, we also analyzed the data in two ways to address the issue of classifying IJs that did not move 
from the introduction point. In this case, S. glaseri following of heterospecifics was only significant $(P \leq 0.05)$ in situations where IJs that did not move were excluded from the analysis (Figure 3 ). While the cumulative probability statistic changed for the other significant assay involving host contact ( $S$. feltiae avoidance of $S$. carpocapsae), significance levels remained at $P<0.05$.

\section{Interspecific Competition}

We tested our third prediction, that following would be associated with competitive ability, by exposing a single host to 50 IJs of each of two species. We scored the outcomes of these interactions in two ways. First, we declared that a species had "won" an individual cadaver if that species produced $90 \%$ or more of the IJs that emerged from the host. Second, proportional IJ production $\left(\mathrm{I}_{\mathrm{p}}\right)$ was calculated as the relative proportion of the number of IJs produced during a mixed-species infection divided by a single-species infection for each species-in this case, an $\mathrm{IJ}_{\mathrm{p}}$ of 0.5 would mean that IJ production in competition was half what it was in a single species infection.

Where "winners" and "losers" were determined, there were clear differences among the pairings. In S. feltiae / S. carpocapsae pairings, $S$. feltiae won in 13 of 15 trials; S. carpocapsae failed to reach the $90 \%$ threshold. In S. feltiae / S. glaseri pairings, S. feltiae won 8 of 15 trials; $S$. glaseri never reached the $90 \%$ threshold. When $S$. glaseri was paired with $S$. carpocapsae, S. glaseri reached the $90 \%$ threshold in 8 out of 13 trials, while $S$. carpocapsae reached $90 \%$ twice; the rest had no winner. From this perspective, we would rank $S$. feltiae as the strongest competitor, and S. glaseri as a stronger competitor than $S$. carpocapsae.

Across heterospecific pairings, there was a reduction in $\mathrm{IJ}_{\mathrm{p}}$ in all cases due to competition; the amount of reduction varied across species pairings (Table 1). In S. feltiae / S. carpocapsae pairings, $\mathrm{IJ}_{\mathrm{p}}$ for $S$. feltiae averaged 0.679 while $S$. carpocapsae averaged 0.027; this difference was statistically significant $\left[t_{(14)}=9.84, P<0.001\right]$. In S. feltiae / S. glaseri pairings, S. feltiae $\mathrm{IJ}_{\mathrm{p}}$ was 0.418 and $S$. glaseri averaged 0.203 ; this difference was not statistically significant $\left[t_{(14)}=-1.29, P=0.218\right]$ (Table 1). In S. glaseri / S. carpocapsae pairings, the $\mathrm{IJ}_{\mathrm{p}}$ of $S$. glaseri was 0.400 while $S$. carpocapsae had an $\mathrm{IJ}_{\mathrm{p}}$ of 0.059 ; this difference was significant $\left[t_{(12)}=4.87, P=0.0004\right]$. This perspective suggests that S. feltiae and S. glaseri are similarly competitive, and that both are stronger competitors than $S$. carpocapsae.

TABLE 1 | Proportional IJ production $\left(I J_{p}\right)$ in various heterospecific pairings.

\begin{tabular}{lcccc}
\hline & \multicolumn{4}{c}{ Mean of relative IJ production (S.E) } \\
\cline { 2 - 5 } Treatment & S. feltiae & S. carpocapsae & S. glaseri & P value \\
\hline S. feltiae-S. & 0.679 & 0.027 & N/A & $<0.0001$ \\
carpocapsae & $(0.062)$ & $(0.010)$ & & \\
S. glaseri-S. & 0.418 & $\mathrm{~N} / \mathrm{A}$ & 0.203 & 0.2183 \\
feltiae & $(0.093)$ & & $(0.043)$ & \\
S. glaseri-S. & N/A & 0.059 & 0.400 & $\mathbf{0 . 0 0 0 4}$ \\
carpocapsae & & $(0.029)$ & $(0.083)$ & \\
\hline
\end{tabular}

N/A, not applicable.

Significant comparisons are highlighted bold.

S.E, standard error.

\section{DISCUSSION}

There are more than 500,000 species of nematodes (Groombridge, 1992; Hodda et al., 2009) that occupy habitats ranging from deep-sea canyons (Vanreusel et al., 2010) to agricultural ecosystems (Yeates and Bongers, 1999). Many species are economically important as causative agents of human diseases, threats to livestock and severe crop pests. Recent research has shown a commonality in the production of and sensitivity to ascaroside pheromones among free-living, plantparasitic, and insect-parasitic nematode species (Choe et al., 2012). Given that these small signaling molecules coordinate development, can have multi-trophic effects (Noguez et al., 2012; Hsueh et al., 2013; Zhao et al., 2016), and affect social behaviors (Hartley et al., 2019; Kaplan et al., 2020; Meng et al., 2020), it follows that they may be involved in trail following, particularly for species where individuals would face challenges to their reproductive success without signals to attract potential mates. However, the implication of ascarosides here is speculative and needs careful analysis of chemicals involved.

Most studies of EPN behavior focus on group responses to stimuli, whereas we focused on how individuals come to form groups. Non-social conspecific aggregations are widespread among animal species and they are generally hypothesized to offer fitness benefits to individuals that would be unavailable if they were solitary (Morrell and James, 2008). Often aggregations are structured based on environmental conditions such as temperature and moisture gradients to which individuals respond similarly (Ozkan-Aydin et al., 2021) or they are driven by individual responses to patchy resources (e.g., Stuart and Gaugler, 1994). These aggregations are by-products of individual responses to external stimuli. Aggregations that form without stimuli associated with resources are exemplified by individuals making decisions to associate with conspecifics, and this relies on communication. Examples of aggregations that result from species-specific signals amongst individuals abound in animals from protists (Mehdiabadi et al., 2006) to chordates (Krause and Tegeder, 1994; Skinner and Miller, 2020). Chemical signals of arthropods are especially well-studied examples of communication leading to aggregative behaviors such as trail following. Prior to our study, no examples were known of following behaviors within the phylum Nematoda. This is particularly surprising given that nematodes often have aggregated population structures and the understanding of the neural bases of aggregation in the model nematode C. elegans (Rogers et al., 2006).

Since following behavior was stronger when the lead nematode was exposed to host cuticle, one could also speculate that molecules associated with the host drives the behavior. Our results do not support the idea that host-associated molecules are the sole driver, since there was not following behavior seen in all heterospecific trials where the lead nematode had been exposed to cuticle, but there was following in all conspecific trials that included host exposure. Further, $S$. feltiae was repelled from lead IJs of $S$. carpocapsae when they were exposed to host cuticle. Additionally, contact with host cuticle has been shown to have dramatic behavioral impacts on those IJs exposed. 
Steinernema glaseri responds to exposure to host cuticle by switching its searching behavior from ranging (which is typically occurs when no host cues are present) to area-concentrated (which signifies the recognition of host cues nearby) (Lewis et al., 1992). Steinernema carpocapsae IJs are significantly more attracted to volatile cues produced by hosts after exposure to G. mellonella cuticle (Lewis et al., 1995b; Baiocchi et al., 2019) and indeed respond most strongly to exposure to the cuticle of potential hosts that support high levels of reproduction (Lewis et al., 1996). Thus, we are confident that changes in the lead IJ as a result of exposure to host cuticle account for following of subsequent conspecific IJs.

Other types of conspecific and heterospecific interactions have similarities to those we describe here. Grewal et al. (1997) studied the attraction of EPN IJs to G. mellonella larvae that were infected by conspecific and heterospecific EPNs $4 \mathrm{~h}$ after exposure to nematodes. In this previous study, S. glaseri, S. carpocapsae and S. feltiae were all included, among other species. Our results reflect the findings of Grewal et al. (1997). Steinernema glaseri IJs were attracted to heterospecific infections, and even insects that were infected with Heterorhabditis bacteriophora, which reflects $S$. glaseri's indiscriminate following of heterospecific IJs. Steinernema feltiae were highly attracted to G. mellonella infected with conspecifics, but their responses were reduced by heterospecific infections compared with their responses to uninfected insects. Steinernema carpocapsae IJs were more attracted to insects with conspecific infections that uninfected insects but were repelled by insects with heterospecific infections. Grewal et al. (1997) suggested that these findings reflected the superior competitive ability of $S$. glaseri within infections. While this could be the case, we show that all sharing of insect cadavers results in a decrease in fecundity.

Many ant and termite species deposit a pheromone trail which serves as a long-lasting orientation cue to individuals of the same species (Traniello, 1982; Reinhard and Kaib, 2001; Fourcassié et al., 2010). Qualitative and quantitative features of trail pheromones change after a forager comes in contact with a resource; the trail pheromone produced by a forager after finding a food source is more attractive to conspecific foragers and remains attractive for a longer time (Traniello, 1982; Reinhard and Kaib, 2001). This situation is analogous to our findings if host contact is substituted for food resources. Host contact may trigger behavioral mechanisms in the lead IJs to recruit followers more efficiently, such as a shift in the composition or quantity of chemical cues that comprise the trail, as suggested by Traniello (1982) and Reinhard and Kaib (2001). We hypothesize that species-specific ascarosides may be involved in the following response of EPN, since they are involved on other EPN behaviors that are related to the formation of aggregations (Shapiro-Ilan et al., 2014, 2019; Hartley et al., 2019; Kaplan et al., 2020).

We measured the fitness cost to EPNs in hosts shared by two species. IJ production was always lower in mixed-species infections than single-species infections, even when there was a clear winner of the competition. We hypothesized that species that were superior competitors would be more likely to follow heterospecific IJs. But only S. glaseri followed heterospecific lead IJs, which is in accord with the recorded cost of sharing a host, and reflects the findings of Grewal et al. (1997), who found S. glaseri to be the most responsive to heterospecific infections. Wang and Ishibashi (1999) found S. carpocapsae to outcompete S. glaseri, but Koppenhofer et al. (1995) observed the opposite, as did we. Perhaps the consistent cost to sharing a host results in selection against individuals following heterospecific IJs compared to those joining monospecific infections.

Overall, our results confirmed our first and second hypotheses, that IJs would follow conspecific IJs, and that their responses would be stronger if the lead IJ had contacted a host. However, we did not find a clear association between the following response to heterospecific IJs and their development in a co-infected host. While EPNs are widely used in biological control in agriculture, there is increasing interest in their value as a model system for understanding animal behavior (Lewis et al., 2006; Griffin, 2012, 2015; Willett et al., 2018), genetics (Duchaud et al., 2003; Ciche and Goffredi, 2007), population biology (Stuart et al., 2006; Griffin, 2015), parasitism (Hallem et al., 2007; Castelletto et al., 2014), mutualism and ecology (Strong et al., 1999; Stock, 2005; Campos-Herrera et al., 2012; Maher et al., 2017). Here, we use EPNs to study group dynamics of a parasite and show for the first time trail following by nematodes. Social interactions are not well-known in this phylum, and this study provides insight into nematode distributions, spatial and temporal population structures, and community dynamics.

\section{DATA AVAILABILITY STATEMENT}

The raw data supporting the conclusions of this article will be made available by the authors, without undue reservation.

\section{AUTHOR CONTRIBUTIONS}

HE designed and conducted the original experiments and collected and analyzed the data in collaboration with KC-G. $\mathrm{HE}$ and KC-G produced the first draft of the manuscript. $\mathrm{KC}-\mathrm{G}$ designed and conducted the experiments and collected and analyzed the data in collaboration with HE. GS helped in experimental design and data analysis, worked with KC-G and HE to develop the experimental arena used for data collection, and contributed to the manuscript editing. DS-I contributed to the experimental design decisions and the manuscript editing. FK contributed to the original experimental design of the experiments, data interpretation, and manuscript editing. HA contributed to the experimental design, data interpretation, and manuscript editing. EL conducted the work in the laboratory and contributed to all phases of data analysis, writing, and editing of the manuscript. All authors contributed to the article and approved the submitted version.

\section{FUNDING}

This work was supported by the United States Department of Agriculture - National Institute of Food and Agriculture, Agriculture and Food Research Initiative (AFRI Grant No. 2018-67013-28064) and the University of Idaho Department of Entomology, Nematology and Plant Pathology. 


\section{REFERENCES}

Alatorrre-Rosas, R., and Kaya, H. K. (1990). Interspecific competition between entomopathogenic nematodes in the genera Heterorhabditis and Steinernema for an insect host in sand. J. Invertebr. Pathol. 55, 179-188.

Ali, J. G., Alborn, H. T., Campos-Herrera, R., Kaplan, F., Duncan, L. W., Rodriguez-Saona, C., et al. (2012). Subterranean herbivore-induced plant volatile increases biological control activity of multiple beneficial nematode species in dinsinct habitats. PLoS One 7:e38146. doi: 10.1371/journal.pone. 0038146

Baiocchi, T., Braun, L., and Dillman, A. R. (2019). Touch-stimulation increases host-seeking behaviour in Steinernema carpocapsae. J. Nematol. 67, 1-8. doi: 10.21307/jofnem-2019-067

Campos-Herrera, R., Barbercheck, M., Hoy, C. W., and Stock, S. P. (2012). Entomopathogenic nematodes as a model system for advancing the frontiers of ecology. J. Nematol. 44, 162-176.

Campos-Herrera, R., Johnson, E. G., El-Borai, F. E., Stuart, R. J., Graham, J. H., and Duncan, L. W. (2011). Long-term stability of entomopathogenic nematode spatial patterns measured by sentinel insects and real-time PCR assays. Ann. Appl. Biol. 158, 55-68.

Castelletto, M. L., Gang, S. S., Okubo, R. P., Tselikova, A. A., Nolan, T. J., Platzer, E. G., et al. (2014). Diverse host-seeking behaviors of skin-penetrating nematodes. PLoS Pathog. 10:e1004305. doi: 10.1371/journal.ppat.1004305

Choe, A., Von Reuss, S. H., Kogan, D., Gasser, B. R., Platzer, E. G., Schroeder, F. C., et al. (2012). Ascaroside signaling is widely conserved among nematodes. Curr. Biol. 22, 772-780.

Ciche, T., and Goffredi, S. (2007). "General methods to investigate microbial symbioses," in Methods for General and Molecular Microbiology, eds C. Reddy, T. Beveridge, J. Breznak, G. Marzluf, T. Schmidt, and L. Snyder (Washington, DC: ASM Press).

Dalzell, J., Kerr, R., Corbett, M., Fleming, C., and Maule, A. (2011). Novel bioassays to examine the host-finding ability of plant-parasitic nematodes. Nematology 13, 211-220. doi: 10.1163/138855410x516760

Dehnhardt, G., Mauck, B., Hanke, W., and Bleckmann, H. (2001). Hydrodynamic trail-following in harbor seals (Phoca vitulina). Science 293, 102-104. doi: 10.1126/science.1060514

Duchaud, E., Rusniok, C., Frangeul, L., Buchrieser, C., Givaudan, A., Taourit, S., et al. (2003). The genome sequence of the entomopathogenic bacterium Photorhabdus luminescens. Nat. Biotechnol. 21, 1307-1313.

Fourcassié, V., Dussutour, A., and Deneubourg, J.-L. (2010). Ant traffic rules. J. Exp. Biol. 213, 2357-2363.

Fushing, H., Zhu, L., Shapiro-Ilan, D. I., Campbell, J. F., and Lewis, E. E. (2008). State-space based mass event-history model i: many decision-making agents with one target. Ann. Appl. Stat. 2, 1503-1522. doi: 10.1214/08-AOAS189

Gehlbach, F. R., Watkins, J. F., and Kroll, J. C. (1971). Pheromone trail-following studies of typhlopid, leptotyphlopid, and colubrid snakes. Behaviour 40, 282294. doi: 10.1163/156853971x00429

Grewal, P. S., Lewis, E. E., and Gaugler, R. (1997). Response of infective stage parasites (Nematoda: steinernematidae) to volatile cues from infected hosts. J. Chem. Ecol. 23, 503-515. doi: 10.1023/b:joec.0000006374.95624.7e

Griffin, C. T. (2012). Perspectives on the behavior of entomopathogenic nematodes from dispersal to reproduction: traits contributing to nematode fitness and biocontrol efficacy. J. Nematol. 44, 177-184.

Griffin, C. T. (2015). "Behaviour and population dynamics of entomopathogenic nematodes following application," in Nematode pathogenesis of insects and other pests, ed. R. Campos-Herrera (Cham: Springer).

Groombridge, B. (1992). "Nematodes," in Global Biodiversity, ed. B. Groombridge (Dordrecht: Springer), 88-92.

Hallem, E. A., Rengarajan, M., Ciche, T. A., and Sternberg, P. W. (2007). Nematodes, bacteria, and flies: a tripartite model for nematode parasitism. Curr. Biol. 17, 898-904. doi: 10.1016/j.cub.2007.04.027

Hartley, C. J., Lillis, P. E., Owens, R. A., and Griffin, C. T. (2019). Infective juveniles of entomopathogenic nematodes (Steinernema and Heterorhabditis) secrete ascarosides and respond to interspecific dispersal signals. J. Invertebr. Pathol. 168:107257. doi: 10.1016/j.jip.2019.107257

Hodda, M., Peters, L., and Traunspurger, W. (2009). "Nematode diversity in terrestrial, freshwater aquatic and marine systems," in Nematodes as environmental indicators, eds M. J. Wilson and T. Kakouli-Duarte (Wallingford: CAB International), 45-93. doi: 10.1079/9781845933852.0045

Hsueh, Y. P., Mahanti, P., Schroeder, F. C., and Sternberg, P. W. (2013). Nematodetrapping fungi eavesdrop on nematode pheromones. Curr. Biol. 23, 83-86. doi: 10.1016/j.cub.2012.11.035

Kaplan, F., Perret-Gentil, A., Giurintano, J., Stevens, G., Erdogan, H., Schiller, K. C., et al. (2020). Conspecific and heterospecific pheromones stimulate dispersal of entomopathogenic nematodes during quiescence. Sci. Rep. 10:5738. doi: 10.1038/s41598-020-62817-y

Kaya, H. K., and Gaugler, R. (1993). Entomopathogenic nematodes. Annu. Rev. Entomol. 38, 181-206.

Koppenhofer, A. M., Kaya, H. K., Shanmugam, S., and Wood, G. L. (1995). Interspecific competition between steinernematid nematodes within an insect host. J. Invertebr. Pathol. 66, 99-103. doi: 10.1890/08-1616.1

Krause, J., and Tegeder, R. W. (1994). The mechanism of aggregation behavior in fish shoals: individuals minimize approach time to neighbors. Anim. Behav. 48, 353-359.

Lawrence, J., Hoy, C., and Grewal, P. S. (2006). Spatial and temporal distribution of endemic entomopathogenic nematodes in a heterogeneous vegetable production landscape. Biol. Control 37, 247-255.

Lewis, E. E., Campbell, J., Griffin, C., Kaya, H., and Peters, A. (2006). Behavioral ecology of entomopathogenic nematodes. Biol. Control 38, 66-79. doi: 10.1016/ j.biocontrol.2005.11.007

Lewis, E. E., Gaugler, R., and Harrison, R. (1992). Entomopathogenic nematode host finding: response to host contact cues by cruise and ambush foragers. Parasitology 105, 309-315. doi: 10.1017/s0031182000074230

Lewis, E. E., Grewal, P. S., and Gauger, R. (1995a). Response of cruiser and ambusher entomopathogenic nematodes (steinernematidae) to host volatile cues. Can. J. Zool. 71, 765-769. doi: 10.1139/z93-101

Lewis, E. E., Selvan, S., Campbell, J. F., and Gaugler, R. (1995b). Changes in foraging behaviour during the infective stage of entomopathogenic nematodes. Parasitology 110, 583-590. doi: 10.1017/s0031182000065306

Lewis, E. E., Ricci, M., and Gaugler, R. (1996). Host recognition behaviour predicts host suitability in the entomopathogenic nematode Steinernema carpocapsae (Rhabditida: steinernematidae). Parasitology 113, 573-579. doi: 10.1017/ s0031182000067627

Maher, A. M. D., Asaiyah, M. A. M., Brophy, C., and Griffin, C. T. (2017). An entomopathogenic nematode extends its niche by associating with different symbionts. Microb. Ecol. 73, 211-223. doi: 10.1007/s00248-016-0 829-2

McMullen, J. G., Peterson, B. F., Forst, S., Goodrich Blair, H., and Stock, S. P. (2017). Fitness costs of symbiont switching using entomopathogenic nematodes as a model. BMC Evol. Biol. 17:100. doi: 10.1186/s12862-017-0939-6

Mehdiabadi, N. J., Jack, C. N., Farnham, T. T., Platt, T. G., Kalla, S. E., Shaulsky, G., et al. (2006). Social evolution: kin preference in a social microbe. Nature 442, 881-882. doi: 10.1038/442881a

Meng, J., Wickham, J. D., Ren, W., Zhao, L., and Sun, J. (2020). Species displacement facilitated by ascarosides between two sympatric sibling species: a native and invasive nematode. J. Pest Sci. 93, 1059-1071.

Morrell, L. J., and James, R. (2008). Mechanisms for aggregation in animals: rule success depends on ecological variables. Behav. Ecol. 19, 193-201. doi: 10.1093/ beheco/arm 122

Mrácek, Z., Kindlmann, P., and Webster, J. A. (2005). Steinernema affine (Nematoda: steinernematidae), a new record for North America and its distribution relative to other entomopathogenic nematodes in British Columbia. Nematology 7, 495-501.

Noguez, J. H., Conner, E. S., Zhou, Y., Ciche, A. T., Ragains, J. R., Butcher, R. A., et al. (2012). A novel ascaroside controls the parasitic life cycle of the entomopathogenic nematode Heterorhabditis bacteriophora. ACS Chem. Biol. 7, 961-966. doi: 10.1021/cb300056q

Ozkan-Aydin, Y., Goldman, D. I., and Bhamla, M. S. (2021). Collective dynamics in entangled worm and robot blobs. Proc. Natl. Acad. Sci. U. S. A. 118:e2010542118. doi: 10.1073/pnas.2010542118

Puza, V., and Mrácek, Z. (2009). Mixed infection of Galleria mellonella with two entomopathogenic nematode (Nematoda: rhabditida) species: steinernema affine benefits from the presence of Steinernema kraussei. J. Invertebr. Pathol. 102, 40-43. doi: 10.1016/j.jip.2009.06.005 
Reinhard, J., and Kaib, M. (2001). Trail communication during foraging and recruitment in the subterranean termite Reticulitermes santonensis De Feytaud (Isoptera, Rhinotermitidae). J. Insect Behav. 14, 157-171.

Rogers, C., Persson, A., Cheung, B., and de Bono, M. (2006). Behavioral motifs and neural pathways coordinating $\mathrm{O} 2$ responses and aggregation in C. elegans. Curr. Biol. 16, 649-659. doi: 10.1016/j.cub.2006.03.023

Ruan, W., Shapiro-Ilan, D., Lewis, E., Kaplan, F., Alborn, H., Gu, H., et al. (2018). Movement patterns in entomopathogenic nematodes: continuous vs. temporal. J. Invertebr. Pathol. 151, 137-143. doi: 10.1016/j.jip.2017.11.010

Shapiro, D. I., and Glazer, I. (1996). Comparison of entomopathogenic nematode dispersal from infected hosts versus aqueous suspension. Environ. Entomol. 25, 1455-1461.

Shapiro-Ilan, D. I., Kaplan, F., Oliveira-Hofman, C., Schliekelman, P., Alborn, H. T., Lewis, E. E., et al. (2019). Conspecific pheromone extracts enhance entomopathogenic infectivity. J. Nematol. 51, 1-5.

Shapiro-Ilan, D. I., Lewis, E. E., and Schliekelman, P. (2014). Aggregative group behavior in insect parasitic nematode dispersal. Int. J. Parasitol. 44, 49-54. doi: 10.1016/j.ijpara.2013.10.002

Skinner, M., and Miller, N. (2020). Aggregation and social interaction in garter snakes (Thamnophis sirtalis sirtalis). Behav. Ecol. Sociobiol. 74:51.

Spiridonov, S., Moens, M., and Wilson, M. (2007). Fine scale spatial distributions of two entomopathogenic nematodes in grassland soil. Appl. Soil Ecol. 37, 192-201.

Stock, P. (2005). Insect-parasitic nematodes: from lab curiosities to model organisms. J. Invertebr. Pathol. 89, 57-66. doi: 10.1016/j.jip.2005.02.011

Strong, D. R., Whipple, A. V., and Dennis, C. B. (1999). Model selection for a subterranean trophic cascade: root-feeding caterpillars and entomopathogenic nematodes. Ecology 80, 2750-2761. doi: 10.1890/0012-9658(1999)080[2750: msfast] 2.0.co;2

Stuart, R. J., Barbercheck, M. E., Grewal, P. S., Taylor, R. A. J., and Hoy, C. W. (2006). Population biology of entomopathogenic nematodes: concepts, issues, and models. Biol. Control 38, 80-102. doi: 10.1016/j.biocontrol.2005.09.019

Stuart, R. J., and Gaugler, R. (1994). Patchiness in populations of entomopathogenic nematodes. J. Invertebr. Pathol. 64, 39-45.

Traniello, J. F. A. (1982). Recruitment and orientation components in a termite trail pheromone. Sci. Nat. 69, 343-345. doi: 10.1371/journal.pone.0090906

Vanreusel, A., Fonseca, G., Danovaro, R., Da Silva, M. C., Esteves, A. M., Ferrero, T., et al. (2010). The contribution of deep-sea macrohabitat heterogeneity to global nematode diversity. Mar. Ecol. 31, 6-20.
Wang, X. D., and Ishibashi, N. (1999). Infection of the entomopathogenic nematode, Steinernema carpocapsae, as affected by the presence of Steinernema glaseri. J. Nematol. 31, 207-211.

White, G. F. (1927). A method for obtaining infective nematode larvae from cultures. Science 66, 302-303.

Willett, D. S., Alborn, H. T., and Stelinski, L. L. (2017). Multitrophic effects of belowground parasitoid learning. Sci. Rep. 7:2067. doi: 10.1038/s41598-01702193-2

Willett, D. S., Alborn, H. T., Stelinski, L. L., and Shapiro-Ilan, D. I. (2018). Risk taking of educated nematodes. PLoS One 13:e0205804. doi: 10.1371/journal. pone.0205804

Yeates, G. W., and Bongers, T. (1999). "Nematode diversity in agroecosystems," in Invertebrate Biodiversity As Bioindicators Of Sustainable Landscapes, ed. M. G. Paoletti (Amsterdam: Elsevier), 113-135. doi: 10.1016/b978-0-444-50019-9. 50010-8

Zhao, L., Zhang, X., Wei, Y., Zhou, J., Zhang, W., Qin, P., et al. (2016). Ascarosides coordinate the dispersal of a plant-parasitic nematode with the metamorphosis of its vector beetle. Nat. Commun. 7:12341. doi: 10.1038/ncomms1 2341

Conflict of Interest: FK is employed by Pheronym, Inc.

The remaining authors declare that the research was conducted in the absence of any commercial or financial relationships that could be construed as a potential conflict of interest.

Publisher's Note: All claims expressed in this article are solely those of the authors and do not necessarily represent those of their affiliated organizations, or those of the publisher, the editors and the reviewers. Any product that may be evaluated in this article, or claim that may be made by its manufacturer, is not guaranteed or endorsed by the publisher.

Copyright (c) 2021 Erdogan, Cruzado-Gutierrez, Stevens, Shapiro-Ilan, Kaplan, Alborn and Lewis. This is an open-access article distributed under the terms of the Creative Commons Attribution License (CC BY). The use, distribution or reproduction in other forums is permitted, provided the original author(s) and the copyright owner(s) are credited and that the original publication in this journal is cited, in accordance with accepted academic practice. No use, distribution or reproduction is permitted which does not comply with these terms. 